\title{
Zulassung von Frauen zum Hochschulstudium (nur) ein erster Schritt
}

\section{Gisela von der Aue}

\section{Senatorin für Justiz des Landes Berlin}

Auch ich begrüße Sie herzlich zur Feier des 60jährigen Bestehens des Deutschen Juristinnenbundes, der nach der aktuellen Satzung das Ziel hat - ich zitiere - „die Wissenschaft durch Fortentwicklung des Rechts zu fördern, unter anderem auf dem Gebiet der Gleichberechtigung und Gleichstellung der Frau in Gesellschaft, Beruf und Familie." Es geht also um Lobbyarbeit, um Mitarbeit an Gesetzesvorhaben, um kritische fachliche Stellungnahmen, um die Fortentwicklung des Rechts. Lassen Sie mich hier aber auch Folgendes hervorheben: Seit Gründung des Deutschen Juristinnenbundes ging und geht es dort vor allem auch darum, die Kontakte der Juristinnen untereinander zu fördern, Netzwerke zu bilden und zu erweitern und Juristinnen zur Wahrnehmung ihrer Interessen anzuspornen.

Diese Interessen haben sich - glücklicherweise, so möchte ich betonen - im Laufe der Jahre verschoben. Um wertschätzen zu können, was wir mit dem Deutschen Juristinnenbund und seiner Vorgängerorganisation, dem bis 1933 bestehenden „Deutschen Juristinnenverein“ schon erreicht haben, lohnt ein Blick in die Vergangenheit, ein Blick auf die frauenpolitische Arbeit von Juristinnen in den letzten neun Jahrzehnten. Ich möchte mich hier dem Anfang des frauenpolitischen Engagements von Juristinnen in der Zeit von 1914 bis 1933 zuwenden. Der 1914 gegründete „Deutsche Juristinnenverein“ hatte noch zum vorrangigen Ziel, die Zulassung von Frauen zu den juristischen Berufen zu erkämpfen. Die juristischen Berufe blieben lange Zeit wie selbstverständlich den Männern vorbehalten und wurden den Frauen vorenthalten. Mit welchen Schwierigkeiten Frauen in der ersten Hälfte des vergangenen

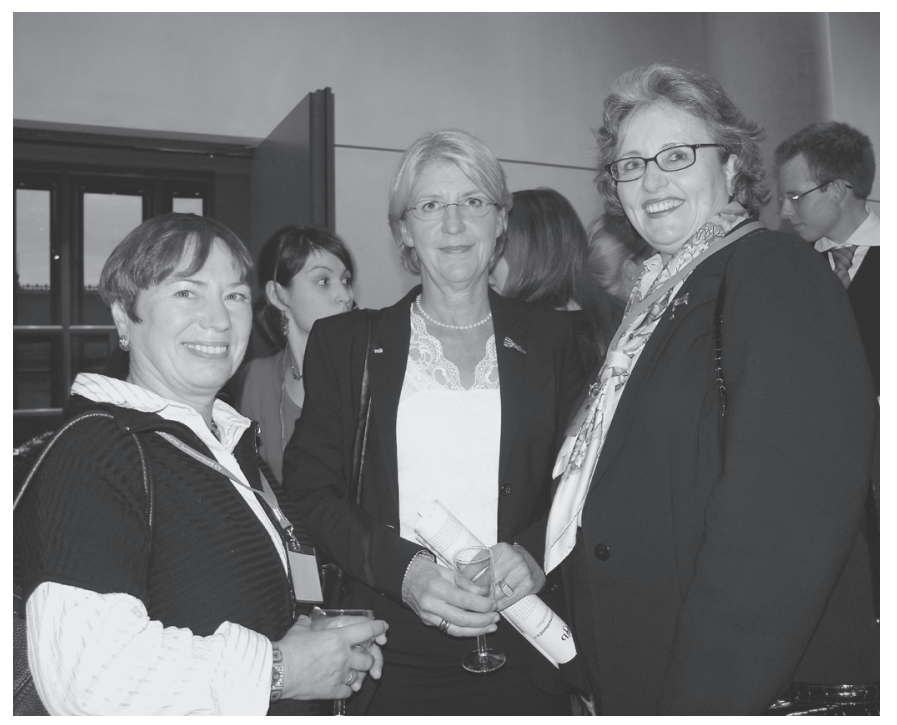

Jahrhunderts zu kämpfen hatten, wenn sie möglichst gleichberechtigt mit den Männern einem juristischen Beruf nachgehen wollten, möchte ich hier am Beispiel einer Frau aus Berlin verdeutlichen, die wie kaum eine andere Frau in den ersten Jahrzehnten des 20. Jahrhunderts gezeigt hat, wie wichtig frauenpolitisches Engagement ist und wie sich Frauen auch unter ungünstigsten Bedingungen durchzusetzen vermögen, wenn sie sich entsprechend organisieren. Ich rede von Marie Munk, der ersten Richterin in Deutschland - gerne erwähne ich hier, dass sie Richterin in Berlin war. Marie Munk, Jahrgang 1885, entstammte einer Berliner Juristenfamilie und studierte $a b$ 1907 in Freiburg, Bonn, Heidelberg und Berlin Rechtswissenschaften. Schon das war zu ihrer Zeit etwas Besonderes. Denn in Preußen hatten - Frau Wagner hat es bereits erwähnt Frauen erst ab 1908 Zugang zu einem Hochschulstudium. Ergänzen kann ich, dass das Großherzogtum Baden mit seinen Universitäten Freiburg und Heidelberg - gemessen an den Maßstäben der damaligen Zeit - fortschrittlich war und schon im Jahr 1900 die Zulassung von Frauen zum Hochschulstudium einführte, was Frau Munk auch nutzte. Die Zulassung von Frauen zum Hochschulstudium war aber nur ein erster Schritt. Denn zunächst, noch bis ins Jahr 1919 hinein, war Frauen auch nach ihrer Zulassung zum Jurastudium die Ablegung der juristischen Staatsprüfungen sowie die Aufnahme in den Referendardienst und damit der Einstieg in die klassischen juristischen Berufe verwehrt. Marie Munk suchte daher nach Abschluss ihres Studiums und einer Promotion nach Alternativen und begann eine Tätigkeit als Assistentin in einer Rechtsanwaltskanzlei. Daneben engagierte sie sich in einer Rechtsberatungsstelle für Frauen. 1919 bestand sie als eine der ersten Frauen überhaupt die erste juristische Staatsprüfung. Im gleichen Jahr trat die Weimarer Reichsverfassung in Kraft, die Frau Wagner hat den maßgeblichen Art. 128 bereits zitiert ausdrücklich bestimmte, dass alle Staatsbürger ohne Unterschied entsprechend ihrer Befähigung und ihren Leistungen zu den öffentlichen Ämtern zuzulassen und dass alle Ausnahmebestimmungen gegen weibliche Beamte zu beseitigen seien. Dessen ungeachtet ordnete noch bis zum Dezember 1922 der maßgebliche preußische Erlass an - ich zitiere: „Zur selbständigen Wahrnehmung der Dienstgeschäft eines Richters, Staatsanwaltes oder Gerichtsschreibers sowie zur Vertretung eines

४ Die Berliner Justizsenatorin Gisela von der Aue, Rechtsanwältin Margret Diwell (Präsidentin des djb von 2001-2005), Präsidentin des Berliner VerfGH, Rechtsanwältin Prof. Asoc. Dr. Jutta Glock (Vors. des Landesverbands Berlin im djb) (v.I.n.r.). 


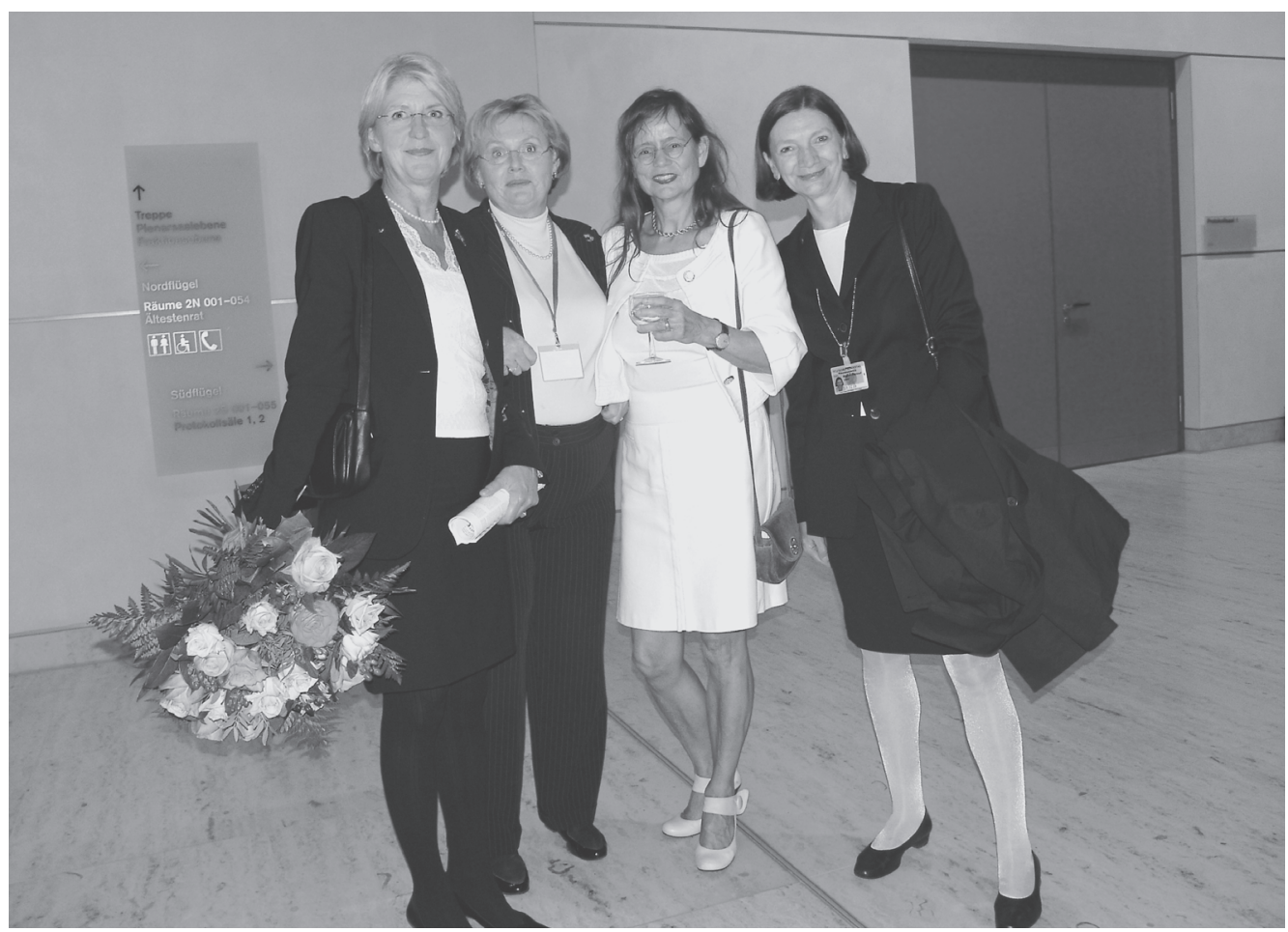

$\varangle$ Rechtsanwältin Margret Diwell (Präsidentin des djb von 2001-2005), Präsidentin des Berliner VerfGH, Richterin am EGMR Dr. h.c. Renate Jaeger, Straßburg, Rechtsanwältin Mechtild Düsing, Vors. der AG Anwältinnen im DAV, Münster, djbPräsidentin Jutta Wagner, Rechtsanwältin, Berlin (v.l.n.r.).

Rechtsanwaltes dürfen Personen weiblichen Geschlechts nicht bestellt werden.“ Wohl gemerkt, dies alles unter Geltung der Weimarer Reichsverfassung, die die Gleichberechtigung von Mann und Frau bereits festschrieb. Noch im September 1922 hatte außerdem die Mehrheit der Mitglieder des Deutschen Anwaltsvereins die Position vertreten, dass die Zulassung von Frauen zu juristischen Berufen eine - ich zitiere wörtlich „Schädigung der Rechtspflege“ bewirke. Zur pseudo-wissenschaftlichen Untermauerung wurden vor allem in den Jahren 1922 und 1923 zahlreiche Aufsätze von Medizinern zur Eignung der Frau für die juristischen Berufe veröffentlicht. So hieß es etwa 1923 im Aufsatz eines Psychologen, dass - ich zitiere erneut - „die Frau wegen ihrer stärkeren Gefühlsbeeinflussung und ihrer ausgeprägten Suggestibilität zu einem streng objektiven Abwägen von Recht und Gesetz ungeeignet“ sei.

Marie Munk ließ sich durch all dies nicht beirren. Sie wurde 1920 Referendarin im Bezirk des Kammergerichts Berlin und legte als erste Referendarin in Deutschland am 7. Januar 1924 die große juristische Staatsprüfung ab. Als erste Frau in Preußen wurde sie schließlich 1924 zur Gerichtsassessorin ernannt und wurde Richterin am Amtsgericht Berlin-Charlottenburg, später am Landgericht Berlin. Damit war sie allerdings lange Jahre in der absoluten Minderheit. Anfang 1933, noch bevor sich die Nazi-Ideologie durchsetzte, nach der die Justiz ohnehin eine reine Männerdomäne zu sein hatte, betrug der Anteil von Frauen unter den Anwälten und Notaren 1,3 Prozent, unter den Richtern und Staatsanwälten sogar nur 0,3 Prozent. Ein wichtiger Grund für den geringen Frauenanteil: Die berufstätigen Juristinnen hatten aufgrund ihrer Minderheitenposition kaum Chancen, die beruflichen Rahmenbedingungen für nachrückende Frauen attraktiv zu gestalten. Damit fehlte die Voraussetzung für einen entscheidenden Faktor bei der beruflichen Entwicklung von Frauen: es fehlte ein berufliches Netzwerk.

Vor allem bei der Frage der Netzwerkbildung sind Organisationen wie damals der Deutsche Juristinnenverein und heute der Deutsche Juristinnenbund unverzichtbar.

Auch dies hat Marie Munk erkannt. Sie war Mitgründerin des Deutschen Juristinnenvereins, von 1923 bis 1933 war sie dessen 2. Vorsitzende. Zusammen mit den anderen Frauen im Deutschen Juristinnenverein betrieb sie mit Nachdruck die Durchsetzung der Zulassung von Frauen zu allen juristischen Berufen, nahm Kontakt mit den vorhandenen weiblichen Abgeordneten im Reichstag auf und stieß so ab 1920 eine lebhafte öffentliche Diskussion zu dieser und zu anderen frauenpolitischen Fragen an. Nachdem Frauen gegen erhebliche Widerstände männlicher Juristen dann grundsätzlich zu juristischen Berufen zugelassen waren, prangerte Frau Munk öffentlich Missstände an, vor allem auch die fehlenden Netzwerke von Frauen und den großen Widerstand gegen berufstätige Frauen unter den männlichen Juristen. Lesenswert ist in diesem $\mathrm{Zu}$ sammenhang ein Aufsatz von Frau Munk in der Juristenzeitung 1925 über praktische Hindernisse für Frauen in der Referendarausbildung.

Schließlich ließ es sich Frau Munk nicht nehmen, Lobbyarbeit zu betreiben und frauenpolitische Themen anzusprechen. Auf dem 33. Deutschen Juristentag in Heidelberg im Jahr 1924 referierte sie neben zwei männlichen Kollegen zur Frage des gesetzlichen Güterstands und präsentierte im Interesse der Frauen bereits Vorschläge zu einer Reform des ehelichen Güterrechts, die in der Tendenz dem entsprachen, was - immerhin gut dreißig Jahre später - 1957 in der Bundesrepublik Deutschland mit Einführung der Zugewinngemeinschaft Ge- 
setz wurde. Aus Anlass dieser Jubiläumsveranstaltung möchte ich schließlich nicht unerwähnt lassen, dass Marie Munk nach dem Zweiten Weltkrieg, als sie bereits in die USA ausgewandert war, dem 1948 gegründeten Deutschen Juristinnenbund verbunden war und beispielsweise auf einer Arbeitstagung des Deutschen Juristinnenbundes im Juni 1950 ein Referat zur Reform des Familienrechts und zur rechtlichen Stellung der Frau in Amerika hielt.

1933 endete dann Frau Munks Engagement zwangsweise. Der Deutsche Juristinnenverein konnte unter der NS-Diktatur nicht mehr weiterarbeiten. Als Jüdin wurde Frau Munk außerdem bereits Mitte 1933 in Vollzug des von den Nationalsozialisten erlassenen Gesetzes zur Wiederherstellung des Berufsbeamtentums aus dem Justizdienst entfernt. Später wäre sie allerdings auch als Frau arischer Abstammung aus dem Justizdienst entlassen worden. Denn ab 1936 durften Frauen in Deutschland nicht mehr als Richterin oder Staatsanwältin arbeiten; ebenfalls ab 1936 wurden Frauen nicht mehr als Rechtsanwältinnen zugelassen. 1939 praktizierten in ganz Deutschland nur noch neun Rechtsanwältinnen; Richterinnen und Staatsanwältinnen gab es gar nicht mehr.

Mit dieser Situation sahen sich die Frauen konfrontiert, die 1948 den Deutschen Juristinnenbund gründeten. Zunächst wurden die verstreut lebenden Juristinnen aufgespürt, erste Netzwerke neu geknüpft und die Juristinnen wieder zur Wahrnehmung ihrer Interessen angespornt. 1960 zählte der Deutsche Juristinnenbund schon knapp 500 Mitglieder. Angesichts der Tatsache, dass es 1960 in der ganzen Bundesrepublik Deutschland nur knapp 350 Richterinnen und Staatsanwältinnen gab - das entspricht einer Frauenquote von drei Prozent - und angesichts eines Frauenanteils von unter zwei Prozent in der Anwaltschaft finde ich diese Mitgliederzahl beachtlich.

Seit 1960 hat sich - gerade auch aufgrund der Arbeit der vielen im Deutschen Juristinnenbund organisierten Frauen einiges zum Positiven gewendet. Lassen Sie mich exemplarisch einen Blick auf die Berliner Justiz werfen. Hier liegt im Jahr 2008 der Anteil der Frauen an allen Richterinnen und Richtern sowie Staatsanwältinnen und Staatsanwälten bei 46,8 Prozent. Vom 1. Juli 2006 bis zum 30. Juni 2008 wurden mehr Frauen als Männer als Richter und Staatsanwälte eingestellt, so dass sich die Frauenquote weiter erhöht hat.

Bemerkenswert ist außerdem, dass Frauen in den herausragenden Leitungsfunktionen in der Berliner Justiz gut vertreten sind. Dies gilt zunächst für die Leitung der Senatsverwaltung für Justiz. Ich begrüße hier herzlich meine Vorgängerinnen im Amt der Senatorin für Justiz, Frau Prof. Jutta Limbach, Frau Lore Maria Peschel-Gutzeit und Frau Karin Schubert. Mit einer etwa vierjährigen Pause zwischen November 1997 und Januar 2002 steht seit März 1989 immer eine Frau an der Spitze der Senatsverwaltung für Justiz. Und auch dies möchte ich betonen: Von den 15 Stellen für Gerichtspräsidenten in Berlin sind aktuell sieben Stellen von Frauen besetzt. In diesem Zusammenhang begrüße ich die fünf hier anwesenden Berliner Gerichtspräsidentinnen: Frau Monika Nöhre, Frau Erna

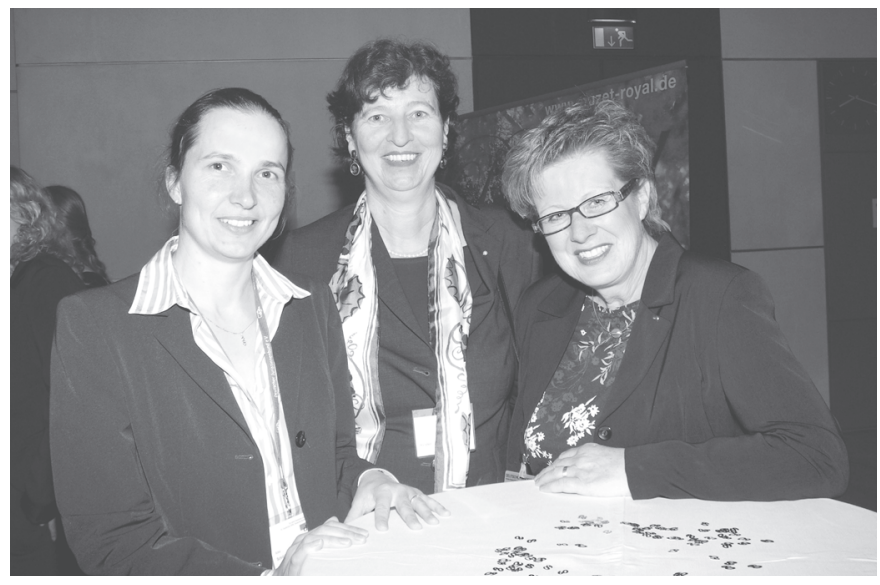

$\triangle$ Die Vorsitzenden des Regionalgruppenbeirats im djb (v.l.n.r.): Gemeinsame Datenschutzbeauftragte Anke Schröder, Loitz, Rechtsanwältin und vereidigte Buchprüferin Birgit Kersten, Oldenburg, und Rechtsanwältin Martina Bosch, Bonn.

Victoria Xalter, Frau Sabine Schudoma, Frau Heike Forkel und Frau Rita Manshausen. Anfang dieses Jahres waren sogar noch neun Gerichtspräsidentenstellen, also deutlich mehr als die Hälfte, mit Frauen besetzt. Zwei Präsidentinnen sind uns nun abhanden gekommen, um andere wichtige Aufgaben zu übernehmen: Frau Karen Buse, bisher Präsidentin des Amtsgerichts Charlottenburg, wird Staatssekretärin in Bremen und Frau Uta Fölster, zunächst Präsidentin des Amtsgerichts Mitte, ist im Frühjahr dieses Jahres zu nichts weniger als zur Präsidentin des Oberlandesgerichts Schleswig ernannt worden. Nicht vergessen möchte ich schließlich, dass an der Spitze unseres Landesverfassungsgerichts mit Frau Margret Diwell auch eine Frau steht. Sie, Frau Diwell, begrüße ich ebenfalls herzlich.

Wir Frauen können uns auf dem bisher Erreichten nicht ausruhen. Ich werde nicht müde, bei unseren Frauenversammlungen in der Berliner Justiz immer wieder zu betonen, wie wichtig Netzwerkbildungen sind. Dies beruht auf meiner Erfahrung, dass Männer, anders als viele Frauen, einerseits schon zahlreiche Netzwerke vorfinden, die über Jahrzehnte gewachsen sind, und dass Männer andererseits die Netzwerkbildung regelmäßig von alleine beherrschen. Deshalb rufe ich immer wieder Frauen dazu auf, sich zu organisieren, untereinander solidarisch zu sein und sich gegenseitig zu ermutigen. Es ist gerade für Frauen wichtig, sich nicht beirren zu lassen und sich nicht von Anfeindungen oder Verunglimpfungen oder durch vermeintlich herrschende gesellschaftliche Vorstellungen vom Weg abbringen zu lassen. Marie Munk, der ich einen großen Teil meines Grußwortes gewidmet habe, kann dafür ein gutes Beispiel sein. Zum Schluss meines Grußwortes möchte ich schließlich den Wunsch äußern, dass der vor 60 Jahren gegründete Deutsche Juristinnenbund auch in den kommenden 60 Jahren einen unverzichtbaren Beitrag zur Bildung von Netzwerken unter den Juristinnen und zur Durchsetzung der Interessen von Juristinnen leistet.

Ich danke Ihnen für Ihre Aufmerksamkeit! 